\title{
Itinéraires Itinéraires
}

Littérature, textes, cultures

2019-1 | 2019

La « renaissance littéraire » africaine en débat

\section{Achille Ngoye et Jean-Roger Essomba : le renouveau du polar africain francophone}

Achille Ngoye and Jean-Roger Essomba: The Revival of the Francophone African Crime Novel

Hervé Tchumkam

\section{OpenEdition}

\section{Journals}

Édition électronique

URL : http://journals.openedition.org/itineraires/5872

DOI : 10.4000/itineraires.5872

ISSN : 2427-920X

Éditeur

Pléiade

Référence électronique

Hervé Tchumkam, « Achille Ngoye et Jean-Roger Essomba : le renouveau du polar africain

francophone », Itinéraires [En ligne], 2019-1 | 2019, mis en ligne le 26 juillet 2019, consulté le 02 août 2019. URL : http://journals.openedition.org/itineraires/5872 ; DOI : 10.4000/itineraires.5872

Ce document a été généré automatiquement le 2 août 2019

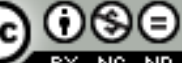

Itinéraires est mis à disposition selon les termes de la licence Creative Commons Attribution - Pas d'Utilisation Commerciale - Pas de Modification 4.0 International. 


\title{
Achille Ngoye et Jean-Roger Essomba : le renouveau du polar africain francophone
}

\author{
Achille Ngoye and Jean-Roger Essomba: The Revival of the Francophone African \\ Crime Novel
}

Hervé Tchumkam

1 Dans son article intitulé «Mayhem at the Crossroads: Francophone African fiction and the Rise of the Crime Novel " (2005), Pim Higginson trace une généalogie du roman policier africain autour d'une thèse centrale selon laquelle la naissance du roman policier en Afrique francophone a modifié les centres d'intérêt et les stratégies esthétiques et ainsi, a reconfiguré l'imaginaire de toute une génération d'écrivains africains. Son article rappelle les caractéristiques principales du genre policier, à savoir « le cadre urbain de l'action, son intérêt pour les notions de classes et d'ethnicité, son exploration des paradigmes normatifs en relation avec le droit, la variation des espaces géographiques et sa célébration du jargon» (Higginson 2005: 163) ${ }^{1}$ et souligne avec justesse que ces éléments jouent un rôle déterminant pour les écrivains, dans la mesure où ils leur permettent de reconfigurer les catégories sociales. En effet, on pourrait considérer que l'émergence du roman policier en Afrique signe l'acte de naissance d'une troisième génération d'écrivains, si l'on s'en tient à la classification de Séwanou Dabla (1986) qui parlait des romanciers de la seconde génération. On parlerait donc d'une troisième génération distincte de celle qui la précédait non pas en raison de la présence ou de l'absence de la notion de violence multiforme, mais précisément dans le support d'expression et de représentation de cette violence: la fiction policière. Mais plus intéressant, au sein de cette troisième génération d'écrivains africains, certains se distinguent par la référence à l'immigration, ce que Pim Higginson a appelé «polar de l'immigration ", mais aussi au surnaturel.

2 Tentant de comprendre l'intérêt soudain des écrivains africains pour le polar, lequel était généralement perçu comme paralittéraire, Pim Higginson introduit la notion de polar de 
l'immigration en soulignant le constat suivant: si les énigmes policières africaines avaient au départ l'Afrique pour espace de prédilection, cet espace de la fiction devient ensuite l'Occident et Paris en particulier, ou il se répartit entre Paris et l'Afrique. Sorcellerie à bout portant (1998) d'Achille Ngoye s'inscrit dans cette catégorie de polar de l'immigration. L'action s'y déroule entre la France et le Congo (ancien Zaïre) et bien que l'essentiel de l'action ait lieu en Afrique, il reste que la France, en tant que point de départ matérialisé par l'aéroport Paris-Charles-de-Gaulle qui ouvre le roman, est le point spatial à partir duquel le message du roman d'Achille Ngoye se construit et sans la référence à laquelle l'itinéraire du protagoniste n'aurait pas de sens. Trois ans avant la parution du roman de Ngoye, Jean-Roger Essomba publiait Les Lanceurs de foudre (1995), un roman auquel la critique ne semble pas avoir prêté grande attention, mais qui est d'autant plus pertinent qu'il emprunte les caractéristiques du polar de l'immigration, les enquêtes se déployant doublement du côté de l'Afrique et de l'Occident. Au moyen d'une lecture de ces deux œuvres, je voudrais mettre en relief l'originalité des romans de cette troisième génération d'écrivains africains, en ce qu'ils associent le polar de l'immigration au paranormal pour finalement faire de leurs œuvres de fiction des espèces de Bildungsroman politique, voire géopolitique. J'entends par là que parallèlement au roman d'initiation, le roman policier africain souligne la nécessité pour le sujet africain de maîtriser le jeu politique comme condition de sa propre survie dans un contexte où l'État organise l'injustice. Pour ce faire, je proposerai donc une approche de ces textes en tant que romans qui transgressent les codes classiques du roman policier occidental et lui ajoutent une empreinte singulière africaine, avec l'irruption d'enquêtes policières qui entremêlent investigations scientifiques et recours à des savoirs paranormaux comme conditions de la tentative de résolution de l'énigme de la mort.

\section{Polar et intrusion du paranormal}

3 Dans Sorcellerie à bout portant, le protagoniste Kizito Sakayonsa retourne dans son pays natal pour essayer de comprendre les circonstances du meurtre de son frère. Ces cadres vont par la suite donner lieu au développement d'histoires intéressantes dans la mesure où elles indiquent une véritable subversion des modèles d'enquêteur et postulent une nouvelle caractérisation du roman d'Achille Ngoye entre esthétique et politique. Dans Les Lanceurs de foudre en revanche, Jean-Roger Essomba donne à lire une histoire d'autant plus palpitante que les enquêtes se démultiplient et ont lieu à la fois dans un pays imaginaire de l'Afrique et dans un espace hors Afrique appelé l'Occident. Au cœur du roman d'Essomba, se trouve une arme du crime qui échappe aux catégories conventionnelles du meurtre: la foudre, dont l'utilisation et la compréhension ne relèvent pas d'une approche cartésienne, mais d'un pouvoir magique. D'emblée, il faut noter que les intrigues de ces deux romans contredisent ou tout au moins indiquent les insuffisances de certaines postures théoriques sur le roman policier en Afrique francophone. Dans Écritures et discours littéraires (1989), Pius Ngandu Nkashama soutient par exemple que l'un des problèmes auxquels se heurte le roman policier en Afrique réside dans le fait qu'il y a en postcolonie incompatibilité entre l'État postcolonial supposé garantir les cadres juridiques nécessaires à une enquête policière et le fait que ce même État est criminel, d'où la mise en avant d'une contradiction néfaste à l'émergence du roman policier en Afrique : 
La fiction romanesque du policier ne peut nullement évoluer sur deux plans contradictoires; à savoir, la quête de la vérité et la dénonciation du système dans lequel est menée cette quête, ou qui fonde le principe de la vérité. (Ngandu Nkashama 1989 : 209)

4 La quête de la vérité et la dénonciation du système dans lequel est mené cette enquête posent problème dans la mesure où il n'y aurait donc pas adéquation entre le but de l'enquête et les institutions supposées la protéger ou en tout cas fonder son principe de validité. Ce point de vue de Pius Ngandu est remarquable en effet, dans la mesure où les systèmes politiques en postcolonie reposent essentiellement sur la dé-liaison de l'État et des citoyens, plutôt que sur la protection de ceux-ci par le premier. C'est à ce titre qu'on pourrait d'ailleurs établir une analogie entre le citoyen africain aux prises avec les pouvoirs postcoloniaux et une figure centrale de la Rome antique: l'homo sacer. En m'appuyant sur la notion de «vie nue » théorisée par Giorgio Agamben dans Homo Sacer. Le pouvoir souverain et la vie nue (1998), je signalerai qu'on peut observer, au moins théoriquement, dans la société africaine contemporaine prise en charge par les romans de Ngoye et Essomba, une mutation intéressante au cœur de laquelle l'homme africain, l' homo africanus, devient littéralement un homo sacer, c'est-à-dire ce citoyen qu'on ne peut mettre à mort sous les formes rituelles mais dont la mort n'est pas considérée comme homicide. La vie du citoyen africain insacrifiable est garantie par son droit à un procès équitable, et pourtant cette même vie est en permanence exposée à la mort. En clair, l'analogie que je perçois entre le citoyen africain face aux pouvoirs politiques et l'homo sacer d'Agamben réside dans le paradoxe entre le caractère sacré de la vie et la mise à mort qui n'est pas considérée comme homicide. Mais là serait un tout autre programme de recherche ${ }^{2}$.

Revenant au roman policier, on peut observer que les itinéraires des personnages comme Kizito Sakayonsa de Sorcellerie à bout portant d'Achille Ngoye, mettent en avant la possibilité de ce "dire " que Pius Ngandu Nkashama estime impossible. Car en effet, le " polar de l'immigration » ou " polar du retour » ne rompt pas les amarres avec l'Afrique qui est le lieu de cristallisation des deux plans contradictoires relevés par le critique. De la même manière, on pourrait tout aussi bien soutenir la thèse selon laquelle Jean-Roger Essomba en alliant la quête de la vérité et la dénonciation des systèmes africains et occidentaux dans lesquels cette quête est menée, réussit à réconcilier ces deux plans contradictoires, en refusant précisément que les enquêteurs de tout bord aient accès à la vérité recherchée et à la punition des coupables. Autrement dit, dans ce cas et dans une logique purement philosophique, la puissance n'est-elle pas supérieure à l'action? La posture de Pius Ngandu Nkashama est valable étant donné le corpus de polars africains existant quand il écrit ses Écritures et discours littéraires. Mais il me semble aussi que sur un plan de théorie littéraire, sa position serait peut-être à nuancer dans la mesure où cette écriture de l'aveuglement qui essaie de «dire» un système indicible tout en reconnaissant son inaptitude à le « dire " pleinement (ceci renverrait à la contradiction que décèle Pius Ngandu Nkashama) confirme justement la potentialité de l'œuvre d'art en Afrique, et notamment de la fiction policière. Car au sens où l'entend Franck Evrard, «si la littérature policière semble donner l'impression de prendre ses distances ou de se remettre en cause, cela vient peut-être de sa contrainte structurelle: rendre problématique le récit du crime, raconter l'impossibilité de raconter ${ }^{3} »$ (1996: 83). L'art deviendrait ainsi le lieu par excellence de l'objectivation de la négation, et ces hypothèses sont vérifiables dans la manière dont les intrigues des polars d'Achille Ngoye et de JeanRoger Essomba sont organisées. 
Sorcellerie à bout portant raconte les mésaventures de Kizito Sakayonsa de retour dans son pays natal pour tenter d'élucider le mystère de la mort de son frère Tsham, officier d'armée décédé dans un accident de voiture, d'après la version officielle donnée par les services de l'armée et de l'État. Un détail frappant annonce la complexité de l'enquête : le corps de Tsham n'aurait jamais été retrouvé et la voiture accidentée aurait mystérieusement disparu. Jusque-là, rien n'annonce un polar singulier, excepté que par une espèce de prolepse, quelques pages plus haut Achille Ngoye introduit le monde du surnaturel dans l'énigme de la mort; alors que le protagoniste de Ngoye relit le télégramme lui annonçant la mort de son frère, le narrateur fait une incursion dans le passé des deux frères et évoque de manière presque anodine le règne du paranormal. D'abord, alors que Kizito est encore dans l'avion qui le ramène dans son pays natal, il est informé par un Britannique de la prégnance à Kinshasa de la sorcellerie « hi-tech », une histoire de «sorcerers [qui] mènent des raids nocturnes aériens dans des avions furtifs [... ], sans le moindre outil de navigation » (Sorcellerie: 17 ). Plus haut dans le texte, parlant de Kizito, le narrateur affirme que l'enfant était un « casse-tête pour les siens lorsqu'il se mit à clopiner du jour au lendemain [et que] les toubibs et guérisseurs ne surent en établir l'origine, tandis qu'un voyant détectait le signe de mauvais augure" (Sorcellerie: 14). L'horizon occulte qui va guider la totalité de l'enquête devient davantage explicite quelques pages plus tard quand le narrateur informe le lecteur qu' " avec la débâcle économique du pays, aggravée par les pillages à répétition, le surnaturel constituait l'ultime recours pour beaucoup [...]. L'occulte et le sournois avaient repris le dessus dans les rapports sociaux » (Sorcellerie : 34). À partir de ce tableau qui encadre et pourrait-on dire prépare l'horizon d'attente du lecteur, l'on constate qu'Achille Ngoye oriente progressivement son projet d'écriture dans un entre-deux entre l'autopsie du politique et le récit d'un crime à élucider. En effet, d'une part le protagoniste doit comprendre les raisons et les conditions de la mort de son frère, et à l'instar de tout enquêteur, démasquer le meurtrier afin que justice soit faite. D'autre part, le récit tout entier se fonde sur une situation politique précise (celle de l'Afrique postcoloniale) en imaginant pourquoi cette société est au bord de l'explosion et bien parfois de l'implosion. Je reviendrai sur ces deux éléments mais il est important de souligner que le roman d'Achille Ngoye « crée son propre monde », un monde qui mime le réel tout en observant une distance avec celui-ci.

7 Le roman de Ngoye entremêle donc deux modalités apparemment contradictoires mais complémentaires: il n'est pas référentiel, c'est-à-dire factuel, mais imaginaire ou fictionnel. Le roman se rapporte au monde réel, mais en plus, il crée un monde qui ne correspond pas toujours exactement au monde extérieur au texte, comme l'écrit Dorrit Cohn au sujet du rapport entre l'œuvre de fiction et le monde qu'il représente :

[...] l'œuvre de fiction crée elle-même, en se référant à lui, le monde auquel elle se réfère [...]. Le caractère non référentiel n'implique pas qu'elle ne puisse pas se rapporter au monde réel, extérieur au texte, mais uniquement qu'elle ne se rapporte pas obligatoirement à lui [...] 1) ses références au monde extérieur au texte ne sont pas soumises au critère d'exactitude; et 2) elle ne se réfère pas exclusivement au monde réel, extérieur au texte. (Cohn $2001: 29-31$ )

La question de l'univers référentiel ainsi établie, je reviens maintenant sur l'intéressante superposition par Achille Ngoye de l'enquête à mener et de l'autopsie des structures politiques en Afrique, qui de ce point de vue ne laisse aucun autre choix au citoyen que la transgression de la norme établie ainsi que le fera Kizito. Ce dernier débarque en effet dans son pays natal et dès l'aéroport, il assiste à des scènes d'abus de pouvoir de la part de 
la police et remarque la militarisation de l'aéroport de Kinshasa. La succession de scènes irréalistes dont Kizito est victime, allant de l'extorsion à l'intimidation et jusqu'au retrait de son passeport alerte notre détective civil postcolonial sur le fait que, comme le rappelait à juste titre Pius Ngandu Nkashama (1989), il y a une contradiction claire entre le système qui est supposé garantir la loi et les transformations de la loi elle-même. Ainsi, la vérité "officielle », c'est-à-dire celle qui est garantie, établie et validée par les institutions étatiques n'est rien d'autre que la mise en texte légale et institutionnelle de la volonté de ceux qui détiennent le pouvoir. En effet, «l'enthousiasme rationaliste, la fascination pour la modernité de la science [...] aboutissent à une sacralisation de la quête de la vérité qui n'interdit pas le recours à la magie, aux sciences occultes [...] L'imaginaire ne peut se contenter toujours du point de vue trop étroitement positiviste ou scientiste » pour reprendre les mots de Franck Évrard (1996: 94). Ce recours aux sciences occultes, le protagoniste d'Achille Ngoye a tant et si bien perçu sa nécessité que son séjour dans son pays va être une véritable descente aux enfers dans le monde de la magie.

Tandis que chez Achille Ngoye le protagoniste revient de la France en Afrique pour se lancer à la recherche des mobiles et responsables de la mort de son frère, avec Jean-Roger Essomba il va s'agir de tout autre chose. Dans un petit village africain, des hommes recourent à la foudre pour réparer les injustices et redonner justice aux hommes et femmes qui ont été lésés par la justice institutionnelle, la justice d'État. Constatant une série de morts de personnes bien ciblées - ce sont des autorités administratives de l'Est du Cazaïbon, pays imaginaire d'Afrique -, les pouvoirs publics décident de mettre leur arsenal policier et juridique en marche pour arrêter les coupables et les mettre hors d'état de nuire. Ce qui est hautement intéressant avec le roman de Jean-Roger Essomba c'est que la justice ne se déploie plus pour rétablir l'ordre social, mais précisément pour protéger les acteurs du renforcement d'un désordre caractérisé par la prédation du peuple par les élites politiques et administratives. Dès le début du roman, on voit un personnage solliciter le pouvoir des lanceurs de foudre afin d'être rétabli dans une justice qui lui a été refusée par un certain Henri Agoumou, le procureur de la République, autrement dit le représentant et garant du droit des citoyens. Le dialogue qui s'installe entre le jeune homme assoiffé de justice et le lanceur de foudre est intéressant dans la mesure où il fournit des éléments d'appréciation de l'univers juridique du pays de référence de la fiction, de même qu'une réflexion pertinente sur les usages de la notion de justice en postcolonie. Face à l'hésitation du lanceur de foudre qui tient à préciser à son interlocuteur que la foudre est « une arme de dissuasion qui est là pour faire régner la justice, la paix et la concorde ", le jeune homme développe une réflexion qui mérite d'être reprise ici en dépit de sa longueur :

Vous dites bien justice! Qu'est-ce que je vous demande, moi, si ce n'est la justice? Je viens de passer injustement trois années de ma vie en prison. Pendant ce tempslà, un autre est parti avec ma femme. Et vous trouvez que ce serait utiliser le feu à tort que de me rendre justice? [...] Je vous en prie, sage Goro, une injustice flagrante a été commise et je veux que justice soit rendue comme l'ont jadis prôné nos ancêtres. (Lanceurs : 11)

De la citation qui précède, deux constats importants s'imposent. D'abord, Les Lanceurs de foudre de Jean-Roger Essomba, un peu à la manière de L'Archer bassari (1984) de Modibo Sounkalo Keita, introduit dans le polar africain une nouvelle modalité du crime dans la mesure où, plutôt que d'occasionner l'instabilité sociale, le crime vient rétablir l'équilibre qui a été brisé par une justice au service des puissants et au détriment du peuple. Mais allant plus loin que Modibo Sounkalo Keita, et c'est le deuxième constat, l'arme du crime 
n'est utilisable et donc intelligible que par un petit groupe d'initiés qui ont un pouvoir surnaturel, d'où l'intrusion du paranormal dans le roman policier africain non pas comme responsable négatif du crime, mais plutôt comme outil de rétablissement de l'équilibre judiciaire et donc social et politique. C'est donc la foudre, nouvelle arme du crime, qui va ôter la vie à Henri Agoumou, procureur de la République, personnage que le roman présente d'ailleurs comme un homme aux ambitions démesurées qui n'avait pas de scrupules, capable de grotesques flagorneries devant ses supérieurs hiérarchiques mais du pire mépris pour ses subalternes, en un mot, un véritable «bras censeur de l'oligarchie, [dont] le nombre d'innocents qui croupissaient dans les prisons par sa faute était impressionnant» (Lanceurs: 19). C'est donc la mort du procureur, suivie de la psychose qui va gagner la ville, qui va décider les autorités administratives du Cazaïbon à confier au commissaire Longbi et à ses inspecteurs de police une mission impossible qui consistera à nettoyer la province de ces lanceurs de foudre, justiciers d'un autre genre.

Il est également notable que parmi les policiers qui vont mener l'enquête des décès par foudre, l'inspecteur qui va infiltrer le village des détenteurs du pouvoir mystique est le fils d'un contrôleur des impôts vraisemblablement véreux qui avait lui-même été tué par la foudre. Essomba réussit là aussi un tour de force en introduisant dans la fiction policière un détective qui ne se saisit pas de l'enquête pour appréhender un éventuel criminel (ce qui correspond à la mission des agents de police, celle de garantir et/ou rétablir la sécurité publique), mais plutôt pour accomplir une vengeance personnelle. Tout ceci éclaire en conséquence le fonctionnement de la justice dans l'Afrique postcoloniale où la notion de sécurité étatique se double de celle d'ambitions personnelles. Alors que les autorités du pays imaginaire de Jean-Roger Essomba s'organisent pour venir à bout des justiciers de la foudre, un groupe d'Occidentaux se prépare, sous le couvert d'organisations humanitaires, à exfiltrer de l'or du pays et à aller à la recherche de la foudre, une arme si puissante qu'elle pourrait leur permettre de devenir les hommes les plus puissants de la planète. En effet, du fait de son intraçabilité "scientifique", la foudre conférerait à qui détient les secrets de son usage plus de pouvoir même que l'arme nucléaire qui constitue, de nos jours, l'arme la plus redoutable qui existe. L'on comprend, en lisant le roman d'Essomba, pourquoi le $\mathrm{D}^{\mathrm{r}}$ Edwell qui est à la tête de l'organisation humanitaire aux visées non avouées qui est résolu à entrer en possession du secret de la foudre, constitue sa propre équipe d'espions qui opèrent sur le continent africain. Ce continent, le Dr Edwell ne le connaît que trop bien car le roman nous apprend qu'il a commencé sa carrière d' ' humanitaire » au Zaïre, le même pays dans lequel Kizito Sakayonsa, le protagoniste d'Achille Ngoye, va faire la rude expérience de la recherche de la vérité après la mort, mieux, l'assassinat de son frère Tsham.

\section{Vers un Bildungsroman politique}

Dans ce Zaïre où le maréchal dictateur qui a dirigé le pays d'une main de fer pendant trois décennies vient d'être détrôné par Laurent Désiré Kabila, la suite des tribulations de Kizito va s'articuler progressivement autour de deux paramètres transgressifs qui valent la peine d'être signalés: d'abord, Kizito en tant qu'enquêteur n'est pas policier de formation, il n'a donc jamais été formé aux rouages d'une enquête policière normale. Ensuite, outre le fait que la police qui est acquise à la cause du pouvoir ne résoudra jamais l'énigme de la mort de Tsham, il faut ajouter que les paramètres qui entourent la disparation du frère de Kizito échappent aux lois de la physique et de la raison, tant les 
détails ésotériques abondent autour de la vie même du major Tsham. Résolu à comprendre les conditions du décès de son frère, Kizito va être par la suite victime de menaces, et face à sa détermination, il va être l'objet de pratiques occultes. Tout se construit dans cet espace colonial soit autour de la violence des pouvoirs politiques, soit autour de la magie. Cette spécificité de la scène du crime rend l'enquête d'autant plus impossible que même les détectives privés que Kizito recrute pour l'aider sont victimes de la nécropolitique ambiante en postcolonie. L'univers social africain postcolonial se singularise, comme dirait Achille Mbembe (2004), par le fait que la politique tend à se constituer uniquement comme une forme de dépense. Ce qui est frappant dans la machine répressive de l'État c'est qu'elle se déploie de manière à compliquer la cartographie du crime. Le détective postcolonial doit donc désormais faire face à deux crimes, l'un étant intimement lié à l'autre. Résoudre l'énigme de la mort dépend d'abord de la résolution de celle de la violence institutionnelle. Ainsi, la somme des faits divers qui ponctuent le roman d'Achille Ngoye prend un sens bien particulier dans la relation que je vais essayer d'interpréter entre l'enquête d'une part et la fiction politique d'autre part. Si l'on admet avec Franck Évrard que «le fait divers constitue davantage un "prétexte" destiné à rendre problématique le réel et à découvrir derrière la mise en scène spectaculaire du fait brut, une réalité complexe qui met en jeu la société et l'idéologie » (1996: 79), il devient on ne peut plus clair que Sorcellerie à bout portant d'Achille Ngoye envisage l'énigme policière tout en ouvrant une autre enquête, celle qui porte sur le système politique africain contemporain.

12 Le politique dans l'Afrique contemporaine, nous rappelle Achille Mbembe, accorde une place centrale à la pratique du pouvoir comme pensée et pratique de guerre. Tout s'y organiserait donc, poursuit le penseur de la postcolonie, autour d'une destruction qui procure jouissance. Mais bien plus, et là réside le lieu significatif du croisement des romans de Ngoye et Essomba, toujours selon Mbembe :

[...] ce travail de destruction comporte deux aspects. Le premier traite de l'extraction/consumation/excrétion des richesses naturelles (or, diamants, et autres produits du sous-sol). Le deuxième consiste, pour l'essentiel, à «donner la mort ». Il est une manifestation éclatante du pouvoir absolu et souverain, que celuici exprime sur le plan de l'intention, de l'acte ou du fantasme. (Mbembe $2004: 152$ )

13 En suivant le fil de la pensée d'Achille Mbembe et en l'appliquant aux fictions d'Achille Ngoye et Jean-Roger Essomba, il ressort que la mission de «surveiller et punir » dont se chargeait l'État moderne d'après Michel Foucault (1975) est devenue, dans le cas de l'Afrique dont il est question, la mise en place d'un état d'exception permanent comme nouveau paradigme de la souveraineté. Plutôt que de s'atteler à l'étude de la permanence du pouvoir qui s'exerce par le contrôle des corps dans le mode d'affirmation du pouvoir souverain en Afrique, il faudrait donc peut-être s'intéresser aux manières dont la spectacularisation de l'État postcolonial rentre également en ligne de compte dans cette manière dont le pouvoir souverain maintient droit de vie et de mort sur les citoyens. Guy Debord est certainement le penseur qui le mieux a cerné cette dimension de ce qu'il appelle la "société du spectacle » dans l'ouvrage du même nom et dont les analyses sont reconsidérées et poursuivies dans ses Commentaires sur la société du spectacle. Dans La Société du spectacle (1967), Guy Debord affirme dès sa quatrième thèse que loin d'être un ensemble d'images, le spectacle est plutôt un rapport social entre des personnes, médiatisé par des images. Une vingtaine d'années plus tard, l'essayiste remarque que le spectacle qui se trouvait au centre s'est renforcé et se trouve désormais au plein cœur de tout rapport social entre l'État et les citoyens. Sorcellerie à bout portant est le théâtre du 
déploiement des techniques de ce que Guy Debord nomme la société du spectacle. Dans sa quête dont l'objet est de comprendre le décès de son frère et d'identifier les responsables de sa mise à mort, Kizito Sakayonsa fait en effet face à un pouvoir qui renforce ses capacités d'exécution de la société spectaculaire par le surnaturel. De ce point de vue, la suspension temporaire des droits des citoyens qui caractérise le justitium du droit romain qui garantissait à l'État ces droits devient une situation dans laquelle Kizito se retrouve en permanence. Qu'il s'agisse des intimidations et des menaces dont il est l'objet, ou des pratiques occultes comme la scène de sorcellerie au cimetière avec ses vêtements, Kizito fait en effet la dure expérience d'un pouvoir qui «insiste sur les grands moyens du spectacle, afin de ne rien dire de leur grand emploi »(Debord [1988] $1992: 18$ ). Tel est en effet le cas lorsque les autorités de la présidence de la République où le défunt frère de Kizito était en service mettent en avant leur pouvoir financier et leur appareil représentatif aux obsèques de leur victime. Ce qu'il faut comprendre c'est que le frère de Kizito, militaire de formation, et grand bourreau au service du pouvoir, avait fini par devenir gênant tant il connaissait les secrets d'un pouvoir qui repose entre autres sur sa dimension mystérieuse. À l'origine de cette situation, il se trouve un aspect de la vie en postcolonie qui échappe d'abord à Kizito dont on voit bien que le retour d'exil n'était pas préparé, et donc ne pouvait être heureux, ainsi que l'a magistralement démontré Ambroise Kom (2002). C'est que, ainsi que le théorise Guy Debord, la société du spectacle débouche sur une fin parodique de la division du travail, c'est-à-dire qu'en contexte africain, "le devenir-monde de la falsification était aussi un devenir-falsification du monde » ([1988] 1992 : 23). Plus clairement, Kizito ignore tous les codes d'interprétation de la société dans laquelle il revient et dont il découvrira qu'il ne sait rien. En effet, et toujours suivant la pensée de Guy Debord, le spectacle organise l'ignorance de ce qui advient et tout de suite après, l'oubli de ce qui a quand même pu en être connu, de sorte que finalement, le plus important est le plus caché. Ainsi, le spectacle déploie la fin de l'histoire par le gommage des traces et l'élimination des preuves comme c'est le cas dans Sorcellerie à bout portant dans lequel « le gouvernement du spectacle, qui à présent détient tous les moyens de falsifier l'ensemble de la production aussi bien que de la perception, est maître absolu des souvenirs comme il est maître incontrôlé des projets qui façonnent le plus lointain avenir» (Debord [1988] 1992 : 23-24). Cela semble d'autant plus vrai dans le cas du roman d'Achille Ngoye que Kizito verra sa famille débarquer à son insu et malgré lui de la France. S'ensuivront un nombre de péripéties et de confrontations directes et indirectes avec la machine du pouvoir politique qui contraindront le protagoniste et sa famille à la fuite clandestine. On comprend donc pourquoi, selon Pim Higginson qui analyse Sorcellerie à bout portant du point de vue du roman noir :

The impenetrability of the plot reflects the complexity of the country; still, the real story is the protagonist Zito's repeated failure to situate himself in a place of vaguely recognizable contours, but whose secret handshakes, political climate, and tortuous capillary flows of power, privilege, and influence he does not understandnot because they are incomprehensible but because he no longer masters his environment ${ }^{4}$. (Higginson $2011: 102$ )

L'incapacité pour l'enquêteur à maîtriser son environnement et donc à mener une investigation sanctionnée par un dénouement positif dont parle Higginson fait également l'originalité du roman de Jean-Roger Essomba, mais sur deux plans parallèles. Sur un premier plan, ce sont les enquêteurs officiels (policiers) du Cazaïbon qui, ne maîtrisant rien aux contours de l'utilisation de la foudre qui donne la mort, sont incapables de cerner les contours de leur enquête au cœur des morts mystérieuses. Aurait-il d'ailleurs 
pu en être autrement lorsqu'on sait que, contrairement à une investigation policière qui rétablirait la sécurité et la quiétude, il s'agit plutôt d'une mise en branle des structures étatiques qui visent à protéger les détenteurs du pouvoir politique qui en abusent? Dans tous les cas, il est clair que l'inaptitude des forces de police à résoudre l'énigme dans Les Lanceurs de foudre signe irrévocablement l'échec du gouvernement du spectacle, dont Guy Debord disait qu'il a les moyens de falsifier la production et la perception. Sur un deuxième plan et peut-être de manière encore plus originale qu'Achille Ngoye dont les personnages non africains - et notamment Peter Thombs qui dirige la société de gardiennage et de renseignement qui devient adjuvant de Kizito dans son enquête - ont une parfaite maîtrise du jeu politique, social et des influences en postcolonie, Jean-Roger Essomba met en fiction un groupe d'Occidentaux qui, malgré des ressources matérielles importantes et des carnets d'adresses particulièrement fournis, ne parviendra pas à triompher sur cette Afrique désormais en proie à la volonté des acteurs étrangers et surtout occidentaux qui bénéficient du silence complice des autorités locales. Une illustration de cette collusion entre les leaders politiques africains et les groupes d'intérêts occidentaux est, chez Jean-Roger Essomba, par exemple matérialisée par le fait que « fort de son expérience et des appuis que lui donnait sa notoriété, [le Dr Edwell] avait mis sur pied une véritable organisation internationale qui, sous le couvert d'œuvres charitables, pillait consciencieusement les pays où elle officiait. Ce pillage se faisait parfois avec la complicité des dirigeants des pays concernés » (Lanceurs : 40).

En introduisant dans sa fiction une telle évocation de cette entente tacite entre gouvernements africains et groupes occidentaux pour nuire aux peuples en les privant de leurs droits élémentaires, Jean-Roger Essomba par le biais de son roman se transforme lui-même en détective des modalités abjectes de gestion des peuples africains et de leurs ressources naturelles. Ce serait peu dire que d'affirmer qu'il permet d'ouvrir une piste d'analyse des rapports de type Françafrique, dont des essayistes comme François-Xavier Verschave $(1998,2004)$ ou plus récemment, des documentaristes à l'instar de Patrick Benquet (2011) ont proposé une remarquable analyse. Mais bien plus qu'une allusion suggestive à la Françafrique, ce que le roman de Jean-Roger Essomba accomplit avec bonheur c'est de suggérer une réflexion sur l'humanitaire en Afrique. Une scène de dialogue dans Les Lanceurs de foudre est intéressante à résumer ici, avant toute interprétation; en effet, alors que le $\mathrm{D}^{\mathrm{r}}$ Edwell se met aux trousses des détenteurs du pouvoir de la foudre avec des intentions inavouées, il est débusqué par l'un d'eux qui ne correspond absolument pas au nègre naif dont étaient friands les administrateurs coloniaux et que continuent à célébrer les spécialistes du droit sélectif de la personne. Il faut rappeler que le prétexte humanitaire que la fondation du $\mathrm{D}^{\mathrm{r}}$ Edwell choisit et met en avant pour se lancer à la chasse à l'arme la plus discrète et la plus puissante du monde est la construction d'une école. Ayant ainsi mis en avant la scolarisation dans la partie est particulièrement sous-développée de ce pays africain imaginaire, les Occidentaux qui vont compliquer l'intrigue du roman passent donc pour des bienfaiteurs que l'État postcolonial célèbre, comme en témoigne l'ensemble de mesures cosmétiques, hygiéniques et protocolaires qui précède la réception officielle de ces " preux chevaliers " de la cause humaine. Seulement, comme je le rappelais plus haut, une scène carnavalesque qui a lieu entre Ikem, un des dépositaires du pouvoir de la foudre et le leader des humanitaires est significative. Alors qu'Ikem laisse entendre que la construction de l'école n'est qu'un prétexte pour une fin insidieuse, le $\mathrm{D}^{\mathrm{r}}$ Edwell s'offusque en ces termes : «Je vous trouve bien injurieux à l'endroit de quelqu'un qui vous 
apporte de l'aide » (Lanceurs : 85). Et la réponse d'Ikem qui ne se fait pas attendre résume bien les matrices cachées de l'aide humanitaire dans les pays dits sous-développés :

De l'aide! Ne me faites pas rire, docteur. Vous apportez quelques instruments pour que ces pauvres enfants apprennent à lire leur misère et à célébrer votre grandeur et vous appelez ça aide? De toutes les façons, je ne veux pas vous juger. Je veux juste vous faire comprendre que j'ai compris votre jeu. (Lanceurs : 85 )

La posture du villageois qui démasque celle de l'Occidental en mission humanitaire est profondément révélatrice de ce qu'il conviendrait de percevoir comme la parade centrale qui préside désormais aux (non) interventions de l'Occident et/ou de ce qu'on appelle la communauté internationale dans les pays en proie aux guerres, parfois doublement réelles et symboliques. Didier Fassin a réfléchi à l'intrusion sur la scène politique des sentiments moraux dans son remarquable essai intitulé La Raison humanitaire à l'intérieur duquel il affirme avec force que «la compassion peut paradoxalement s'avérer un sentiment qui permet de faire l'économie d'une action plus exigeante» $(2010: 230)$. En ramenant cette affirmation au contexte du roman de Jean-Roger Essomba, on pourrait dire en effet que la leçon décapante que l'intrigue policière enseigne c'est précisément que les pouvoirs politiques locaux, en appuyant, en célébrant et surtout en étant complices actifs ou passifs des missions humanitaires, contribuent à mettre en avant l'émotionnel, le pathos plutôt que la raison ou la logique. En d'autres termes, par sa prise de position sur la problématique de l'aide, Ikem le lanceur de foudre du roman d'Essomba devient symboliquement un détective qui livre les conclusions d'une enquête au cœur de la mise à mort physique ou symbolique d'un peuple réduit à sa pure vie biologique, sans aucune médiation. En jouant ce rôle problématique, les États postcoloniaux en Afrique participent donc consciemment à l'occultation des causes sociales de la misère des peuples, et du même fait renforcent la permanence des vies précaires que Judith Butler a analysées en se servant du concept de "visage» développé par Emmanuel Lévinas. L'analyse de la philosophe dans l'essai intitulé Vie précaire correspond bien à la forme que prennent ces vies précaires dans Les Lanceurs de foudre, en ceci que le roman de JeanRoger Essomba invite à s'interroger sur le fait qu'« il se fait que d'autres prétendent avoir des droits moraux sur nous, nous adressent des exigences morales, que nous n'avons pas sollicitées et que nous ne sommes pas libres de refuser » (Butler 2005 : 164).

17 À la fois chez Didier Fassin et chez Judith Butler, il y a la mise en relief de ces vies qui ne comptent pas, celles des citoyens soumis à l'injonction d'acceptation d'un ordre mondial humanitaire inique et destructeur. C'est au nom de ces vies précaires que parle Ikem dans le roman de Jean-Roger Essomba, qui souligne le fait que l'action humanitaire qui occasionne la présence des Occidentaux dans son village est le résultat d'une exigence morale imposée aux villageois, et que ces derniers n'ont pas demandé, et qu'ils ont encore moins le droit de refuser. L'humanitaire en Afrique, suggère le roman policier, serait aussi une modalité du " donner la mort » en postcolonie en prétextant « sauver la vie ", étant entendu que les dimensions affective et émotionnelle mises en avant dans les logiques humanitaires bloquent précisément la voie aux droits inaliénables dont doivent bénéficier les peuples africains. Si ces peuples en Afrique sont généralement sans défenses face au rouleau compresseur de l'État local et du gouvernement mondial, dans les romans policiers d'Achille Ngoye et Jean-Roger Essomba, ils sont dotés d'un pouvoir de résistance impressionnant.

Dans Sorcellerie à bout portant par exemple, face à la démission du gouvernement et à son incapacité ou son refus de rendre une justice équitable, on note tour à tour le rôle de la 
justice privée et notamment la justice populaire (lynchages ou encore «supplice du pneu »), la nécessité de tuer comme unique condition de survie (Kizito venu au Zaïre pour élucider une mort est contraint de donner la mort pour ne pas mourir lui-même), ainsi que les règlements de compte purs et simples (le frère de Kizito aurait été appâté et tué par Malesso qui vengeait ainsi le meurtre de sa famille par les troupes du maréchal dictateur alors sous le commandement du major Tsham). Dans Les Lanceurs de foudre, la revanche du peuple s'appuie sur la maîtrise d'une arme paranormale, la foudre, qui fait de ses utilisateurs des sortes de détectives qui n'ont pas besoin de mener une enquête mais qui sont aussi doublement des bourreaux, non pas du peuple, mais simplement de quiconque porte atteinte à ou enfreint la justice. De manière presque carnavalesque, le roman de Jean Roger Essomba renverse les structures classiques de l'investigation policière et inscrit le meurtre dans une dimension transnationale.

Les victimes de la foudre qui répare les injustices dont ont été frappées les pauvres populations du Cazaïbon se comptent non seulement parmi les tenants du pouvoir dans ce pays d'Afrique postcoloniale, mais aussi en Occident où tous les apôtres du mal sont punis et sanctionnés par la mort que même la police occidentale, avec tout son arsenal scientifique et technique, ne parvient pas à expliquer ou à comprendre rationnellement. L'univers de la fiction devient ainsi un véritable carnaval, à comprendre au sens Bakhtinien :

Le carnaval est un spectacle sans rampe ni division entre interprètes et spectateurs. Dans le carnaval tout le monde participe activement, tout le monde communie au jeu carnavalesque. Le carnaval ne s'observe pas ni même, à proprement parler, ne se joue, il se vit, c'est-à-dire qu'on y vit d'une vie carnavalesque. Et la vie carnavalesque est une vie tirée de son cours ordinaire, dans une certaine mesure une «vie à l'envers », un «monde renversé » («le monde à l'envers»). (Bakhtine $1970: 143-144)$

20 En effet, en échappant au contrôle de la police officielle, la nature des crimes par la foudre dans le roman de Jean Roger Essomba abolit les limitations de la vie ordinaire et brise tout pouvoir et toute situation hiérarchique. Le pouvoir est désormais contrôlé par le petit peuple qui, à l'inverse des gouvernements postcoloniaux et de leurs complices occidentaux, signe le changement et le renouveau des rapports entre les catégories sociales de la vie ordinaire. Le carnaval à l'œuvre ici serait bien celui qui, dans l'esprit de Bakhtine, «n'érige rien en absolu mais proclame la joyeuse relativité de toutes choses » (1970: 146), ainsi que l'atteste le fait que le pouvoir de la foudre revient finalement à Clara Ellison, personnage non africain mais, et c'est ce qui importe, sensible au fait que la charité sans justice n'est rien d'autre que le maintien de l'injustice. Deux constats s'imposent au terme de la lecture de Sorcellerie bout portant d'Achille Ngoye et Les Lanceurs de foudre de Jean-Roger Essomba.

Premier constat : ces textes représentatifs du roman policier africain se situent en dehors du canon du roman policier occidental sur plusieurs plans. D'abord, le propre du polar étant de réduire l'altérité de la violence à une signification, ces romans n'y parviennent pas et se limitent à suggérer des pistes sur lesquelles je reviendrai ci-dessous. Ensuite, le policier n'est plus un détective formé aux rouages du droit civil et pénal ainsi qu'aux méthodes d'enquête, il devient n'importe qui et parfois, il peut même être un sorcier. Puis l'irruption de la sorcellerie dans le polar et l'introduction du surnaturel dans un genre classiquement régi par la rationalité devient un mode de transgression majeur du modèle du roman policier. En cela, Achille Ngoye et Jean-Roger Essomba se servent de l'imaginaire pour remettre en question la primauté de la thèse selon laquelle il existe un 
seul modèle de rationalité, ainsi que l'avaient déjà fait en leur temps des philosophes africains à l'instar de Fabien Eboussi Boulaga (1977)et Meinrad Pierre Hebga (1998).

Second constat : se servant du genre policier en tant que genre perçu comme étant le plus proche de la réalité en raison de son ancrage populaire, Achille Ngoye tout comme JeanRoger Essomba réussissent en effet un tour de force remarquable. L'enquête qui se situe au cœur de la fiction et qui est compliquée par des données surnaturelles permet aux écrivains camerounais et congolais de proposer en effet un Bildungsroman politique qui se veut très différent du modèle du Bildungsroman allemand.

Le Bildungsroman classique se définit généralement comme la transformation d'un jeune homme en adulte mature, et cette transformation met en relief la société de l'évolution du personnage comme lieu de l'avènement d'un homme d'expérience, au contraire du jeune homme qu'il était au départ ${ }^{5}$. Au cœur du Bildungsroman se trouve donc une fonction rigoureusement sociale, c'est-à-dire l'intégration du personnage en situation de formation. Or, ces critères ne correspondent en rien à la trajectoire cependant très initiatique de Kizito le protagoniste d'Achille Ngoye par exemple. Au contraire, tout indique que si Sorcellerie à bout portant, au-delà de son intrigue policière, se donne à voir comme une radioscopie de l'Afrique postcoloniale, Les Lanceurs de foudre pour sa part peut être lu comme un anti-Bildungsroman occidental en raison de son originalité sur deux points : la variation dans le statut du héros et la finalité de la formation, qui n'est plus celle d'un jeune homme en adulte mature, mais celle d'un corps social à la fois international et africain qui est mis en garde quant à ce que l'on pourrait considérer comme la finalité du Bildung classique, à savoir l'accès au pouvoir individuel, social ou matériel. En introduisant donc le polar de l'immigration ou les enquêtes au pays natal pour parodier le titre d'un polar de Driss Chraïbi, les romanciers africains modifient ainsi le statut du personnage principal qui ne vient plus nécessairement de la province vers Paris, mais bien souvent de l'Occident vers l'Afrique.

Ensuite, plutôt que de se focaliser sur la mise en avant du lieu de formation du personnage central, les romans de Jean-Roger Essomba et d'Achille Ngoye fournissent des éléments pour naviguer dans le monde politique en Afrique postcoloniale et dans son prolongement planétaire, au moyen d'un éclairage sans détours sur la responsabilité que partagent dirigeants africains et groupes de pression internationaux sur la déliquescence de l'Afrique. La société africaine à l'intérieur de laquelle se déroule l'enquête étant encore plus mystérieuse que le meurtre lui-même, la mise en avant du détective postcolonial de Sorcellerie à bout portant, de même que la déroute des détectives officiels et occidentaux dans Les Lanceurs de foudre, apportent tout de même un savoir sur son monde référentiel. Ce savoir n'est rien d'autre que le fait que, ainsi que l'écrit Guy Debord: «On croyait savoir que l'histoire était apparue, en Grèce, avec la démocratie. On peut vérifier qu'elle disparaît du monde avec elle » ([1988] 1992 : 36). De ce fait, étant par ailleurs entendu que "partout où règne le spectacle, les seules forces organisées sont celles qui veulent le spectacle» (Debord [1988] 1992: 37), on peut émettre l'hypothèse que par le choix de paramètres poétiques atypiques tels que l'intrusion dans la fiction du surnaturel comme actant, le roman policier africain subvertit le genre de l'intérieur et en transgresse les règles. Cette transgression a une conséquence plus importante sur la fonction de la littérature de manière générale. Je suggère en effet que la spectacularisation de la mort dans la fiction policière africaine serait une forme subtile de "résistance » en ce sens qu'en réponse au déploiement excessif et irresponsable d'un pouvoir qui se fonde sur la « société du spectacle » et la prolifération systématique de la mort, le sujet postcolonial 
décide de le défier en lui opposant des réactions à leur tour spectaculaires, mais à l'envers parce que non réductibles à une interprétation rationnelle ou scientifique.

En d'autres termes et pour conclure provisoirement, les écrivains de polars africains, par le biais de leurs personnages, ont arraché au pouvoir la capacité d'usage exclusif de la mort que ce pouvoir avait capturé. Cette permanence de la mort inexplicable serait peutêtre aussi la forme africaine d'une «insurrection qui vient » et avec laquelle l'État devra composer ou s'anéantir, tant il est vrai avec Giorgio Agamben que «la profanation de l'improfanable est la tâche politique de la génération qui vient» (2005: 122). En définitive, en mettant en avant polar d'immigration, paranormal (surnaturel) et Bildungsroman politique, Achille Ngoye et Jean-Roger Essomba se servent du genre policier pour effectivement remplir deux fonctions essentielles qu'il faut indiquer en conclusion. La première, à savoir la fonction sociale et pédagogique du roman policier en Afrique, Sorcellerie à bout portant l'inscrit dans la problématique des retours d'exil et fonctionne comme une mise en garde « en direction des Africains qui séjournent trop longtemps en Occident en oubliant de se tenir au courant des évolutions que subissent les pays du continent. La qualité du retour, suggère [Ngoye], est étroitement liée à la maîtrise du terrain et de la psychologie des acteurs en place» (Kom 2002). Ce faisant, le roman d'Achille Ngoye rend possible l'autopsie des pouvoirs postcoloniaux qui illustrent à souhait le constat selon lequel «la nouveauté de la politique qui vient, c'est qu'elle ne sera plus une lutte pour la conquête ou le contrôle de l'État, mais une lutte entre l'État et le non-État (l'humanité)» (Agamben 1990: 88). La seconde fonction du roman policier africain, qui est la fonction philosophique, est remarquablement illustrée par le roman de Jean-Roger Essomba. En effet, Les Lanceurs de foudre autorise et invite à une réflexion à la fois sur l'humanitaire et sur le totalitarisme local (africain) et mondial en ceci qu'il met à nu les logiques capitalistes et impérialistes qui installent durablement les citoyens africains dans l'insécurité et la terreur. En raison de quoi il ne reste plus qu'à conclure cette réflexion avec ces mots de Jean Marie Teno dans son documentaire Le Malentendu colonial (2004) : «Combattre l'injustice afin que la charité devienne inutile.» Sortir des logiques coloniales qui ont systématisé le «don de la mort » et remplacer la raison humanitaire par le droit des peuples et la justice, telle semble être en effet l'ultime leçon à tirer du roman policier africain qui associe polar de l'immigration, paranormal et Bildungsroman politique.

\section{BIBLIOGRAPHIE}

Agamben, Giorgio, 1990, La Communauté qui vient, Paris, Seuil.

Agamben, Giorgio, 1997, Homo Sacer. Le pouvoir souverain et la vie nue, Paris, Seuil.

Agamben, Giorgio, 2005, Profanations, Paris, Payot, Rivages.

Bakhtine, Mikhaïl, 1970, Problèmes de la poétique de Dostoïevski, Lausanne, Éditions L'Âge d'Homme.

Benquet, Patrick, 2011, Françafrique : 50 années sous le sceau du secret, Compagnie des Phares et

Balises, 159 min, DVD.

Itinéraires, 2019-1 | 2019 
Bresnick, Adam, 1998, « The Paradox of Bildung: Balzac's Illusions Perdues », Modern Language Notes , vol. 113, no 4, p. 823-850.

Butler, Judith, 2005, Vie précaire, Paris, Éditions Amsterdam.

Cohn, Dorrit, 2001, Le Propre de la fiction, Paris, Seuil.

Debord, Guy, 1967, La Société du spectacle, Paris, Buchet-Chastel.

Debord, Guy, [1988] 1992, Commentaires sur la société de spectacle, Paris, Gallimard.

Dabla, Sewanou, 1986, Nouvelles écritures africaines. Romanciers de la Seconde Génération, Paris, L'Harmattan.

Eboussi Boulaga, Fabien, 1977, La Crise du Muntu : authenticité africaine et philosophie, Paris, Présence africaine.

Essomba, Jean-Roger, 1995, Les Lanceurs de foudre, Paris, L'Harmattan.

Évrard, Franck, 1996, Lire le roman policier, Paris, Dunod.

Fassin, Didier, 2010, La Raison humanitaire, Paris, Gallimard, Seuil.

Foucault, Michel, 1975, Surveiller et punir : naissance de la prison, Paris, Gallimard.

Hebga, Meinrad Pierre, 1998, La Rationalité d'un discours africain sur les phénomènes paranormaux, Paris, L'Harmattan.

Higginson, Pim, 2005, « Mayhem at the Crossroads: Francophone African Fiction and the Rise of the Crime Novel », Yale French Studies, n 108 , p. 160-176.

Higginson, Pim, 2011, The Noir Atlantic. Chester Himes and the Birth of the Francophone African Crime Novel, Liverpool, Liverpool University Press.

Kom, Ambroise, 2002, « Il n'y a pas de retour heureux », Mots Pluriels, n² 20, [En ligne], http:// motspluriels.arts.uwa.edu.au/MP2002ak.html, consulté le 11 juillet 2019.

Mbembe, Achille, 2004, « Essai sur le politique en tant que forme de la dépense », Cahiers d'études africaines, $\mathrm{n}^{\circ}$ 173-174, [En ligne], http://journals.openedition.org/etudesafricaines/4590, consulté le 11 juillet 2019 .

Ngandu Nkashama, Pius, 1989, Écritures et discours littéraires, Paris, L’Harmattan.

Ngoye, Achille, 1998, Sorcellerie à bout portant, Paris, Gallimard.

Sounkalo Keita, Modibo, 1984, L'Archer bassari, Paris, Karthala.

Tchumkam, Hervé, 2016, « Homo Africanus, Homo Sacer. Biopouvoir et ethnoracialisation des rapports sociaux en France », Terroirs, vol. 1, n² 2, p. 117-135.

Verschave, François-Xavier, 1998, La Françafrique : le plus long scandale de la République, Paris, Stock.

Verschave, François-Xavier, 2004, De la Françafrique à la mafiafrique, Bruxelles, Tribord.

\section{NOTES}

1. «Its urban setting and preoccupation with class and ethnicity; its exploration of normative paradigms through the trope of the law; its liminal literary status; its evolution 
through geographic displacement; and its celebration of the vernacular.» (Nous traduisons).

2. Pour une lecture d'une interprétation du sujet africain et diasporique comme homo sacer, voir Hervé Tchumkam (2016).

3. Je souligne.

4. «Le caractère hermétique de l'intrigue est à l'image de la complexité du pays; la véritable histoire est celle de Zito, le protagoniste, qui ne parvient pas à retrouver ses repères, et à qui tout échappe, des jeux d'influences aux manœuvres de pouvoir, non pas parce qu'ils sont incompréhensibles, mais simplement parce qu'il ne maîtrise plus l'environnement dans lequel il opère. »

5. Lire à ce propos Adam Bresnick (1998).

\section{RÉSUMÉS}

Dans l'histoire littéraire africaine, qu'il s'agisse du roman colonial ou du roman de la révolte solitaire, le roman africain a toujours été assimilé au roman réaliste. Cependant, au tournant des années 1980, on a observé dans la production littéraire africaine un tournant significatif marqué par le dépassement du roman réaliste et l'avènement de la fiction policière. Cette transgression des frontières génériques s'est accompagnée de l'émergence de nouvelles thématiques, au rang desquelles l'intrusion dans le roman policier du paranormal et de la sorcellerie. Le présent article se propose de lire Sorcellerie à bout portant (1998) d'Achille Ngoye et Les Lanceurs de foudre (1995) de Jean-Roger Essomba comme deux romans qui renouvellent le genre policier en Afrique francophone. Au moyen d'une lecture qui se situe au point de rencontre entre esthétique et politique, mon objectif sera de montrer comment ces fictions associent polar de l'immigration et paranormal. Je suggérerai finalement que ces romans interrogent la place de l'Afrique et de l'Africain dans le partage du sensible, ouvrant ainsi sur la mise en évidence d'une nouvelle forme de ce que j'appelle Bildungsroman politique propre au contexte africain.

In African literary history, whether in the colonial novel, the family romance, or the novel of solitary revolt, the African novel has generally been considered realistic. However, in the early eighties new genres and authors challenged the reign of the realistic novel, giving birth to the African detective novel. Some of the crime novels in Africa have renewed aesthetics by introducing sorcery and the paranormal into the plots. To that effect, this article offers a reading of Achille Ngoye's Sorcellerie à bout portant and Jean-Roger Essomba's Les Lanceurs de foudre as novels that recast Francophone African crime fiction. By underlining the intersection between politics and aesthetics in these novels, my aim will be to highlight the ways in which these novels bring together witchcraft and immigration. Ultimately, I will contend that this revival of African fiction seeks to interrogate the place of Africa and Africans in the distribution of the sensible, thus hinting at the coming-of-age of a new form of what I term the political Bildungsroman that is specific to the African context. 
INDEX

Mots-clés : Ngoye (Achille), Essomba (Jean-Roger), paranormal, polar, Bildungsroman politique

Keywords : Ngoye (Achille), Essomba (Jean-Roger), sorcery, crime fiction, political

Bildungsroman

\section{AUTEUR}

HERVÉ TCHUMKAM

Southern Methodist University 